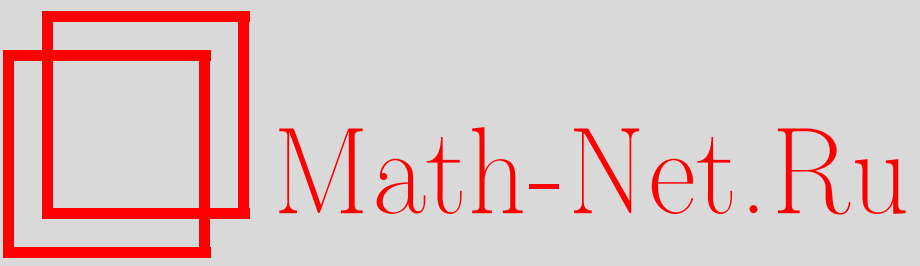

А. И. Эстеров, Плотности чисел Бетти множеств меньших значений квазипериодических функций, УМН, 2000, том 55, выпуск 2, 157-158

DOI: https://doi.org/10.4213/rm286

Использование Общероссийского математического портала Math-Net.Ru подразумевает, что вы прочитали и согласны с пользовательским соглашением

http://www.mathnet.ru/rus/agreement

Параметры загрузки:

IP: 3.91 .87 .62

26 апреля 2023 г., 16:19:59 


\title{
ПЛОТНОСТИ ЧИСЕЛ БЕТТИ МНОЖЕСТВ МЕНЬШИХ ЗНАЧЕНИЙ КВАЗИПЕРИОДИЧЕСКИХ ФУНКЦИЙ
}

\author{
А.И. ЭСТЕРОВ
}

Для гладкой функции $f: M \rightarrow \mathbb{R}^{1}$ обозначим через $M_{c}$ множество меньших значений $\{x \in M \mid$ $f(x) \leqslant c\}\left(c \in \mathbb{R}^{1}\right)$. Для почти любой функции $f$ на замкнутом многообразии $M$ (т.е. для любой функции кроме множества бесконечной коразмерности в пространстве всех функций) топология множества $M_{c}$ (в частности, его числа Бетти) меняется только при проходе $c$ через одно из конечного числа критических значений функции $f$. Для периодической (имеющей $k$ линейно независимых периодов) функции $f: \mathbb{R}^{k} \rightarrow \mathbb{R}^{1}$ числа Бетти $b_{j}\left(M_{c}\right)$, вообще говоря, бесконечны. Нетрудно видеть, что для почти любой периодической функции существует предел

$$
\beta_{j}(c)=\lim _{v \rightarrow \infty} \frac{b_{j}\left(B_{v}^{k} \cap M_{c}\right)}{V\left(B_{v}^{k}\right)},
$$

где $B_{v}^{k} \in \mathbb{R}^{k}$-замкнутый шар радиуса $v$ с центром в нуле, а $V\left(B^{v}\right)$ - объем этого шара. Величина $\beta_{j}(c)$ также меняется только при прохождении одного из конечного числа критических значений функции $f$.

Пусть теперь $f$ - гладкая квазипериодическая функция.

ОПРЕДЕЛЕНИЕ 1. Квазипериодической функчией называется композищия $f=\phi \circ P \circ L$ линейного мономорфизма $L: \mathbb{R}^{k} \rightarrow \mathbb{R}^{n}$, естественной факторизации $P: \mathbb{R}^{n} \rightarrow T^{n}$ и гладкой функции $\phi: T^{n} \rightarrow \mathbb{R}^{1}$ при условии, что образ $P \circ L$ всюду плотен в $T^{n}$.

Функция $f$ имеет, вообще говоря, бесконечное количество критических значений, которые как-то распределены в $\mathbb{R}^{1}$. В некоторых моделях хаоса (см. [1]) возникает вопрос о существовании предела (1) и о характере его зависимости от $c$. Существование предела типа (1) для эйлеровой характеристики множества $M_{c}$ и его кусочная гладкость как функции от с были доказаны в [2]. Здесь доказьвается следующее утверждение.

ТЕОРема 1. Для почти любой квазипериодической функции $f$ предел (1) существует при любом с и непрерывно зависит от с.

В формулировке теоремы “для почти любой квазипериодической функции $f$ ” означает “для почти любой функции $\phi: T^{n} \rightarrow \mathbb{R}^{1}$, определяющей $f^{\prime \prime}$.

ОПРЕДЕЛЕниЕ 2. Исчезающим циклом критической точки $x_{0}$ индекса $\alpha$ функции $f$ назовем сфиеру

$$
S^{\alpha-1}=\left\{x \in M \mid u_{1}^{2}+\cdots+u_{\alpha}^{2}=\delta ; u_{\alpha+1}=\cdots=u_{k}=0\right\}
$$

для достаточно малого $\delta$, где $u_{1}, \ldots, u_{k}$ - координаты в окрестности $U$ точки $x_{0}$, в которых $f$ имеет вид $f\left(x_{0}\right)-u_{1}^{2}-\cdots-u_{\alpha}^{2}+u_{\alpha+1}^{2}+\cdots+u_{k}^{2}$.

Исчезающий цикл точки $x_{0}$ не гомологичен нулю в $U \cap M_{f\left(x_{0}\right)} \backslash\left\{x_{0}\right\}$, но может быть гомологичен нулю во всем $M_{f\left(x_{0}\right)} \backslash\left\{x_{0}\right\}$.

ОПРЕДЕЛЕНИЕ 3 . Назовем радиусом обнуления $r\left(x_{0}\right)$ критической точки $x_{0}$ минимальное $v$, при котором исчезающий цикл критической точки $x_{0}$ гомологичен нулю внутри $B_{v}^{k} \cap M_{f\left(x_{0}\right) \backslash}$ $\left\{x_{0}\right\}$ (если такого $v$ не существует, положим $r\left(x_{0}\right)$ равным бесконечности).

Теперь можно сформулировать два утверждения, из которых следует теорема 1.

Теорема 2. Для почти любой квазипериодической функции при любом $c, j \in\{0, \ldots, k\}$ u $r \in[0, \infty]$ существует конечная плотность критических точек индекса $j$ с радиусом обнуления, меньшим $r$, лехащих в $M_{c}$. Она непрерьвно зависит от с. То же самое верно и для плотности критических точек индекса $ј$ с бесконечным радиусом обнуления, лежащих в $M_{C}$. 
Теорема 3. Плотность $\beta_{j}(c)$ равна разности плотностей точек индекса $j$ с конечным радиусом обнуления и индекса $j+1$ с бесконечным радиусом обнуления, лежащих в $M_{c}$ (поэтому она существует для почти любой квазипериодической функции $f$ u непрерывно зависит от $c$ ).

ДоКАЗАТЕЛЬСтво тЕОРЕмы 2 использует следующие факты, содержащиеся в [2]. Пусть $L: \mathbb{R}^{k} \rightarrow \mathbb{R}^{n}$ - линейный мономорфизм и $P: \mathbb{R}^{n} \rightarrow T^{n}$ - естественная факторизация, причем образ $P \circ L$ всюду плотен. Пусть $M^{n-k}$-подмногообразие в $T^{n}$, трансверсальное к образу $P \circ L$. Тогда отношение стандартных форм объема на $\mathbb{R}^{n}$ и $\mathbb{R}^{k}$ задает ориентацию и форму объема $\omega$ на $M^{n-k}$

ОПРедЕЛЕнИЕ 4. Интеграл формы $\omega$ по $M^{n-k}$ называется трансверсальныцм обвемом $M^{n-k}$ и обозначается $\widetilde{V}\left(M^{n-k}\right)$ (трансверсальный объем всегда положителен).

Tеорема 4. Пусть $K=(P \circ L)^{-1}\left(P \circ L\left(\mathbb{R}^{k}\right) \cap M^{n-k}\right)$ - дискретное подмножество в $\mathbb{R}^{k}$. Если трансверсальный обвем $M^{n-k}$ конечен, то существует и равна этому обвему плотность множества $K: \widetilde{V}\left(M^{n-k}\right)=\lim _{v \rightarrow \infty} \frac{\#\left(K \cap B_{v}^{k}\right)}{V\left(B_{v}^{k}\right)}$, где \# обозначает количество точек в множестве, $B_{v}^{k} \subset \mathbb{R}^{k}-$ иар радиуса $v$ с центром в нуле, $а V\left(B^{v}\right)$ - обгем этого иара.

Пусть $M_{j}^{n-k}$ - множество точек $x \in T^{n}$, для которых ограничение $d f$ на подпространство $L \circ P\left(\mathbb{R}^{k}\right) \subset T_{x} T^{n} \cong \mathbb{R}^{n}$ равно нулю, а ограничение $d^{2} f$ на это подпространство является невырожденной квадратичной формой индекса $j$. Существование плотностей, указанных в формулировке теоремы 2 , следует из применения теоремы 4 к множеству $M_{j}^{n-k}$ и его открытым подмножествам $M_{j, r}^{n-k}$, заданным условиями $r(x)<r$. Эти плотности равны соответственно $\widetilde{V}\left(M_{j, r}^{n-k} \cap M_{c}\right)$ и $\widetilde{V}\left(M_{j}^{n-k} \cap M_{c}\right)-\widetilde{V}\left(M_{j, \infty}^{n-k} \cap M_{c}\right)$. Почти любая $\phi: T^{n} \rightarrow \mathbb{R}^{1}$ не является константой ни на каком открытом подмножестве в $M_{j}^{n-k}$, и поэтому для почти любой квазипериодической функции $f$ указанные выше объемы непрерывны как функции от $c$.

ДокаЗАтельство теоремы 3. Рассмотрим $N_{v}=B_{v}^{k} \cap M_{c}(f)$, где $B_{v}^{k} \in \mathbb{R}^{k}$-шар радиуса $v$ с центром в нуле. Из стандартных рассуждений теории Морса (примененных к многообразию с краем) следует, что число Бетти $b_{j}\left(N_{v}\right)$ равно $K_{1}-K_{2}+d$, где $K_{1}$ - число критических точек $x$ индекса $j$, исчезающие циклы которых гомологичны нулю в $N_{v} \backslash\{x\} ; K_{2}$ - число критических точек $x$ индекса $j+1$, исчезающие циклы которых не гомологичны нулю в $N_{v} \backslash\{x\} ; d$ ограничено по модулю суммарной кратностью вырожденных критических точек функции $f$ в $N_{v}$ и критических точек ограничения $\left.f\right|_{\partial B_{v}}$ этой функции на границу шара. Отношение $d / V(B v)$ стремится к нулю для почти любой функции $f$, так как суммарная кратность вырожденных точек конечна для почти любой функции $f$, а суммарная кратность критических точек $\left.f\right|_{\partial B_{v}}$ растет пропорционально $(k-1)$-мерному объему сферы $\partial B_{v}$. Из теоремы 2 следует, что $K_{1} / V\left(B_{v}\right)$ и $K_{2} / V\left(B_{v}\right)$ стремятся к плотностям критических точек индекса $j$ с конечным и индекса $j+1 \mathrm{c}$ бесконечным радиусом обнуления соответственно, лежаших в $M_{c}$ (когда эти плотности существуют). Переход к пределу при $v \rightarrow \infty$ в равенстве $b_{j}\left(N_{v}\right) / V\left(B_{v}\right)=K_{1} / V\left(B_{v}\right)-K_{2} / V\left(B_{v}\right)+d / V\left(B_{v}\right)$ дает утверждение теоремы 3 .

\section{СПИСОК ЛИТЕРАТУРЫ}

[1] Заславский Г.М., Захаров М. Ю., Сагдеев Р.З., Усиков Д. А., Черников А. А. // Письма в ЖЭТФ. 1986. Т. 44. № 7. С. 349-353. [2] Гусейн-Заде С. М. // Функц. анализ и его прил. 1989. Т. 23. № 2. С. 55-56.

Московский государственный

Принято редколлегией университет им. М. В. Ломоносова 26.01.2000 